\title{
Eficiencia de un inhibidor verde extraído de la cáscara de la sandía en la corrosión del acero estructural A36 evaluado en medios ácido y salino
}

\author{
Juan Felipe Rodríguez ${ }^{1 *}$; Estefannia Rodríguez; Luisa Fernanda Suárez ${ }^{1,2}$; \\ Karen Tatiana Velasco ${ }^{1}$; Camilo Ramos ${ }^{1}$; Eduard Malagón ${ }^{1}$ \\ ${ }^{1}$ Escuela de Ingeniería de Materiales, Universidad del Valle (Univalle), calle $13 \mathrm{n} \cdot{ }^{\circ} 100-00$, Cali, Colombia \\ ${ }^{2}$ Grupo de Películas Delgadas, Departamento de Física, Universidad del Valle (Univalle), calle $13 \mathrm{n} .{ }^{\circ} 100-00$, \\ Cali, Colombia \\ *Juan.rodriguez.patino@correounivalle.edu.co
}

Fecha recepción: noviembre 7 de 2019 Fecha aceptación: diciembre 16 de 2019

\section{Resumen}

La corrosión del acero al carbono es uno de los problemas más comunes en industrias tales como las de petróleo, gas y producción de energía, donde, además de los medios agresivos, se utilizan diferentes sustancias corrosivas como ácidos [1]. El uso de inhibidores de corrosión se emplea comúnmente y es la forma más económica para proporcionar protección temporal contra la corrosión. Muchos compuestos sintéticos han mostrado buena actividad anticorrosiva. Sin embargo, la mayoría de ellos son altamente tóxicos para los seres humanos y el medio ambiente [2]. Es por ello que hoy en día se investigan e implementan productos naturales como agentes anticorrosivos [3]. En esta investigación se obtiene y evalúa un extracto a partir de la cáscara de la sandía (Citrullus lanatus) como inhibidor de corrosión del acero estructural $\mathrm{A} 36$ en medio ácido $(\mathrm{HCl} 0,5 \mathrm{M})$ y medio salino $(\mathrm{NaCl} 3,5 \%)$ a diferentes tiempos de exposición (0,11 y 24 días). El comportamiento a la corrosión se estudió a partir de pérdida de peso y métodos electroquímicos, como la extrapolación Tafel. Adicionalmente, se realizó un análisis metalográfico de las muestras expuestas a los medios con y sin inhibidor. La estructura se analizó microestructuralmente a través de observación microscópica. Finalmente, se determinó la eficacia del inhibidor.

Palabras clave: Inhibidor; Corrosión; Sandía; Acero al Carbono. 


\title{
Efficiency of a green inhibitor extracted from the watermelon peel in the corrosion of structural steel A36 evaluated in acid and saline media
}

\begin{abstract}
Carbon steel corrosion is one of the most common problems in industries such as oil, gas and energy production, where in addition to aggressive media, different corrosive substances are used as acids [1]. The use of corrosion inhibitors is specifically used and is the most economical way to provide temporary protection against corrosion, many synthetic compounds have demonstrated good anticorrosive activity. However, most of them are highly toxic to humans and the environment [2]. That is why, today natural products are investigated and implemented as anticorrosive agents [3]. In this investigation, an extract is obtained and evaluated from the sand shell (Citrullus lanatus) as a corrosion inhibitor of structural steel A36 in acid medium $(0.5 \mathrm{M} \mathrm{HCl})$ and saline medium $(3.5 \% \mathrm{NaCl})$ at different exposure times $(0,11$ and 24 days). Corrosion behavior was studied from weight loss and electrochemical methods such as Tafel extrapolation. In addition, a metallographic analysis of the samples exposed to the media with and without inhibitor was performed. The structure was analyzed microstructurally through microscopic observation. Finally, the efficacy of the inhibitor was determined.
\end{abstract}

Keywords: Inhibitor; Corrosion; Watermelon; Carbon Steel.

\section{Eficiência de um inibidor verde extraído da casca de melancia na corrosão do aço estrutural A36 avaliado em meio ácido e salino}

\begin{abstract}
Resumo
A corrosão do aço carbono é um dos problemas mais comuns em indústrias como a produção de petróleo, gás e energia, onde, além de meios agressivos, diferentes substâncias corrosivas são usadas como ácidos [1]. O uso de inibidores de corrosão é especificamente utilizado e é a maneira mais econômica de fornecer proteção temporária contra a corrosão; muitos compostos sintéticos demonstraram boa atividade anticorrosiva. No entanto, a maioria deles é altamente tóxica para os seres humanos e para o meio ambiente [2]. É por isso que hoje os produtos naturais são investigados e implementados como agentes anticorrosivos [3]. Nesta investigação, um extrato é obtido e avaliado a partir da casca de areia

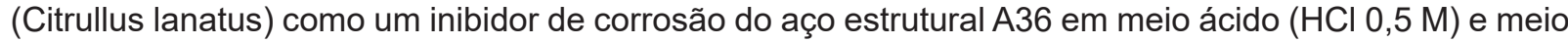
salino ( $\mathrm{NaCl} 3,5 \%$ ) em diferentes tempos de exposição (0, 11 e 24 dias). O comportamento da corrosão foi estudado a partir da perda de peso e métodos eletroquímicos, como a extrapolação de Tafel. Além disso, foi realizada uma análise metalográfica das amostras expostas aos meios com e sem inibidor. A estrutura foi analisada microestruturalmente através de observação microscópica. Finalmente, a eficácia do inibidor foi determinada.
\end{abstract}

Palavras-chave: Inibidor; Corrosão; Melancia; Aço Carbono. 


\section{Introducción}

El empleo de materiales metálicos facilita las condiciones actuales de vida de las personas; sin embargo, la mayoría de los materiales metálicos tiene una elevada susceptibilidad para corroerse [1]. Esto se traduce en grandes pérdidas económicas en los países, mientras que la prevención o la disminución de la velocidad del proceso de corrosión genera importantes inversiones [2].

Uno de los principales métodos para combatir la corrosión es el uso de inhibidores, sustancias que se utilizan en bajas concentraciones en el medio corrosivo, disminuyen o previenen la reacción entre el metal y el medio [3] [4]. Los inhibidores de origen orgánico-sintético son eficientes, no obstante, su naturaleza química los hace persistentes, poco o nulamente degradables, y, en la mayoría de los casos altamente tóxicos [1].

Debido al empleo de estos inhibidores, se ha tenido un impacto ambiental, por lo cual se han buscado nuevas alternativas que reduzcan la velocidad de corrosión de los materiales metálicos y que, además, resulten amigables con el ambiente [1]. Ha cobrado importancia el empleo de polvos, aceites esenciales, infusiones acuosas y extractos naturales obtenidos de las hojas de plantas, cáscaras, frutos, semillas y raíces, como inhibidores ecológicos de la corrosión, debido a su efecto seguro, uso práctico, bajo costo y por ser materiales renovables [5].

\section{Materiales y métodos}

El desarrollo experimental se dividió en dos partes: en la primera parte se efectuaron la extracción y preparación del inhibidor verde, a partir de la cascara de la sandía en forma de una suspensión liquida; y la segunda parte consistió en la evaluación de dicho inhibidor, mediante técnicas electroquímicas, gravimetría e inspección visual. Estas técnicas permiten evaluar el desempeño y mecanismo de inhibición de corrosión de los componentes de la cascara de sandía. Para la obtención del inhibidor fue seleccionada una sandía en buen estado, de la cual se tomó la corteza para iniciar con la preparación del extracto. Siguiendo el procedimiento experimental citado por Lorena M. Saavedra Navarrete [6], se procedió a pesar y licuar $150 \mathrm{~g}$ de cáscara de sandía, sin ningún tratamiento previo, y $800 \mathrm{ml}$ de agua destilada; finalmente, se realizó una filtración retirando los sólidos remanentes de la cáscara, y se obtuvo la parte líquida. Debido a que los inhibidores de corrosión comúnmente son añadidos en pequeñas cantidades, y siguiendo el estudio de María J. Berrocal et al. [7], el inhibidor se añadió en un $10 \%$ del total de la solución.

Las muestras de acero A36 con dimensiones aproximadas de $2,5 \times 2,5 \times 0,3 \mathrm{~cm}$ fueron introducidas en soluciones de $\mathrm{HCl}$ con inhibidor, $\mathrm{HCl}$ sin inhibidor, $\mathrm{NaCl}$ con inhibidor y $\mathrm{NaCl}$ sin inhibidor. Las edades que se usaron para la recolección de datos fueron 0,11 y 24 días; se realizó toma de pérdida de peso, y luego se hicieron las pruebas electroquímicas de extrapolación de curvas Tafel, con el fin de determinar las velocidades de corrosión de las muestras en presencia y ausencia del inhibidor; una vez terminada cada prueba electroquímica, las muestras fueron observadas con ayuda del estereoscopio, con el objetivo de conocer visualmente qué cambios habían sufrido las muestras expuestas a los dos ambientes corrosivos, haciendo una comparación con edades anteriores o con la edad 0 . Finalmente, por medio de los análisis cualitativos realizados en SEM se buscaron determinar los mecanismos de inhibición presentes en la superficie del acero estructural A36 para una edad de 24 días.

\section{Resultados y discusión}

En la figura 1 se muestran los resultados de la inspección visual realizada después de sumergir las muestras en los medios corrosivos de estudio durante 11 y 24 días de exposición. Se logra evidenciar que las muestras expuestas al medio salino con inhibidor, tanto durante 11 como durante 24 días, presentan presentan menor cantidad de productos de corrosión y menor deterioro superficial que las muestras expuestas en este mismo medio sin presencia de inhibidor para los mismos tiempos de exposición. Por otro lado, las muestras inmersas en $\mathrm{HCl}$ sin inhibidor, tanto a 11 como a 24 días evidencian un gran deterioro superficial y mayor presencia de productos de corrosión, características que son más evidentes en la muestra que estuvo expuesta mayor tiempo; además, se puede evidenciar que esta muestra presenta corrosión localizada.

En la figura 2 se evidencia la pérdida de peso en función del tiempo de inmersión para los medios a) $\mathrm{NaCl}$ y b) $\mathrm{HCl}$, respectivamente. Se puede observar que la pérdida de peso de las muestras aumenta con el tiempo de inmersión; este aumento es más significativo en las muestras que se encontraban en el medio sin presencia del inhibidor en ambos casos de estudio, lo que indica que el inhibidor sí cumplió con su función y retrasó la velocidad de corrosión en ambos medios. 
a

- 11 días sin inhibidor

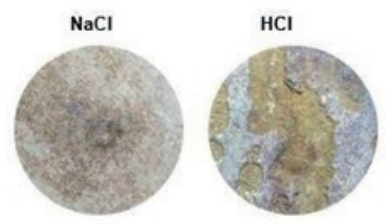

- 11 días con inhibidor $\mathrm{NaCl}$
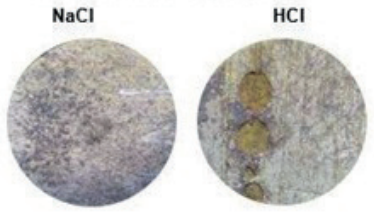

b

- 24 días sin inhibidor

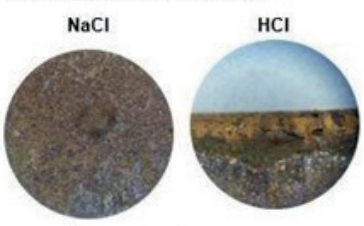

- 24 días con inhibidor

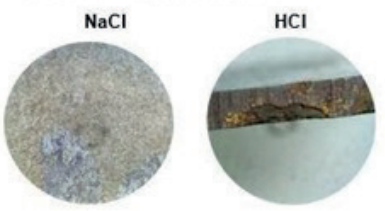

Figura 1. Inspección visual de las muestras sometidas a los medios de estudio a) 11 días de exposición y b) 24 días de exposición.

a

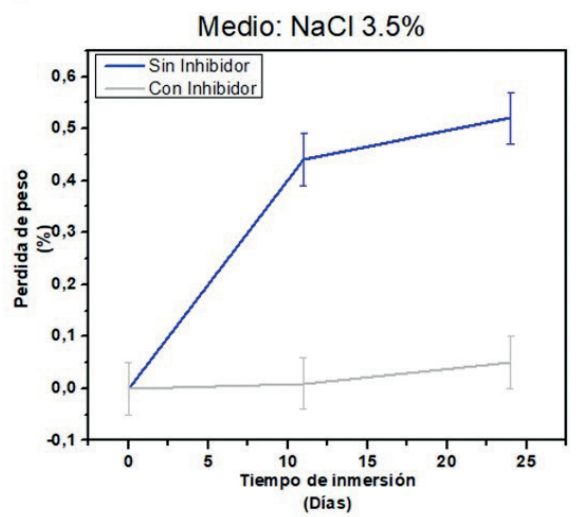

b

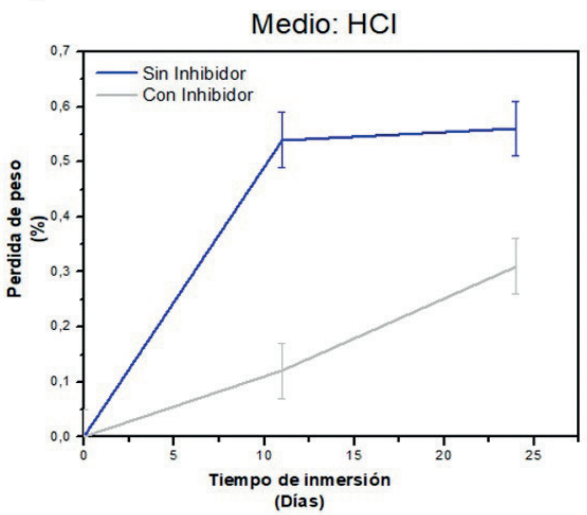

Figura 2. Pérdida de peso para los medios de estudio a) salino y b) ácido.

Se realizó un estudio de microscopía electrónica de barrido (SEM) a las muestras de acero estructural A36 sometidas a los dos ambientes corrosivos durante 24 días con y sin presencia de inhibidor; se puede observar en la figura 3 que tanto las muestras con y sin inhibidor en medio ácido presentan deterioro superficial y productos de corrosión con respecto a la muestra patrón; estos efectos son más agresivos y evidentes en la muestra sin inhibidor expuesta en este medio. Además, se evidencia la formación de una capa no homogénea en la superficie de la muestra expuesta al medio ácido con presencia de inhibidor, la cual pudo servir de protección a la corrosión frente a la agresividad del medio. De manera similar ocurre con las muestras expuestas al medio salino, donde se puede observar un mayor deterioro en la superficie del material en la muestra que se sometió al medio sin presencia del inhibidor. En este caso, la película protectora formada sobre la superficie de la muestra sometida al medio con presencia de inhibidor es más densa y homogénea.

En las figuras 4 y 5 es posible observar el comportamiento de las curvas Tafel de las muestras sometidas en el medio ácido con y sin inhibidor y salino con y sin inhibidor, respectivamente, durante 24 días de exposición. Se logra evidenciar que el comportamiento del inhibidor en ambos medios es de carácter anódico, ya que se puede observar un desplazamiento del potencial de corrosión hacia valores más pasivos, y una disminución de la densidad de corriente (desplazamiento de la curva hacia la izquierda) con respecto a la curva patrón, lo cual concuerda con los resultados obtenidos de las pruebas de pérdida de peso y SEM, donde se evidencia la formación de una capa pasiva sobre el metal respecto a la muestra patrón, la cual ayuda a aislarlo del medio; estos resultados están acordes a la literatura [7]. 


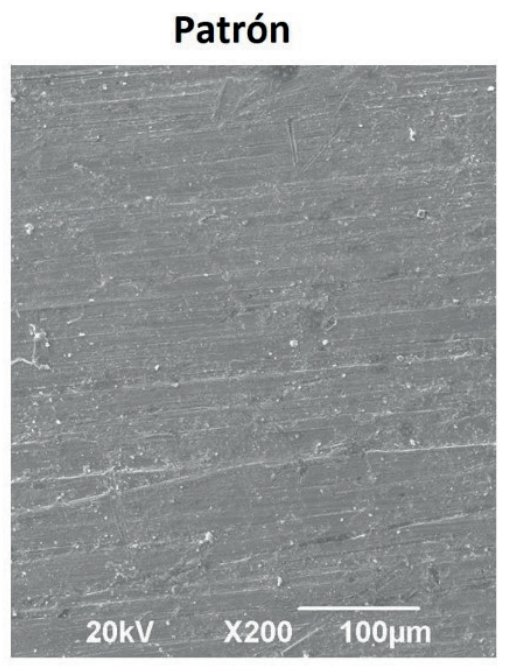

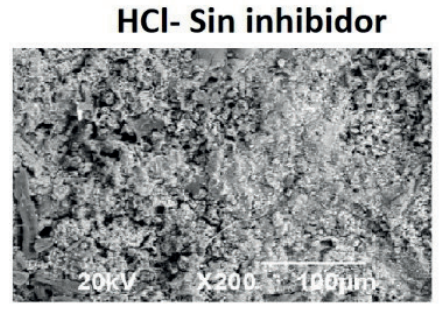

$\mathrm{HCl}$ - Con inhibidor

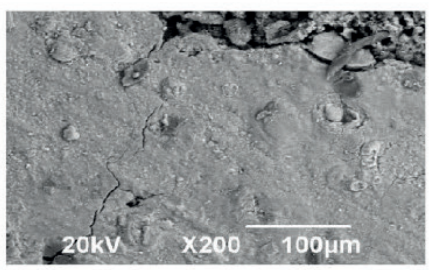

$\mathrm{NaCl}-\mathrm{Sin}$ inhibidor

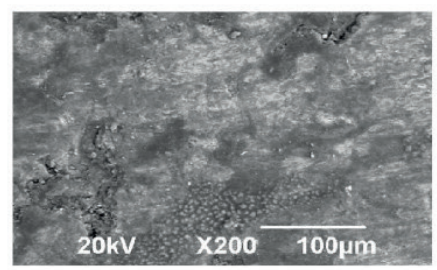

$\mathrm{NaCl}$ - Sin inhibidor

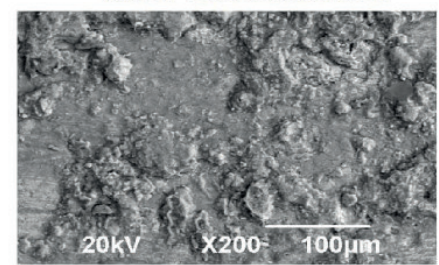

Figura 3. Micrografías SEM de las muestras expuestas durante 24 días en medio ácido y salino con y sin inhibidor.

Comparando el efecto del inhibidor extraído de la cáscara de sandía en los dos medios, resulta evidente que el mejor comportamiento se presenta en el medio salino, como era de esperarse, porque, aunque es un medio agresivo, no lo es tanto como el medio ácido. Por otro lado, el mecanismo de acción de los inhibidores de carácter anódico consiste en formar una capa pasiva sobre la superficie del metal aislándolo del medio; esta capa posiblemente no se formó por completo en

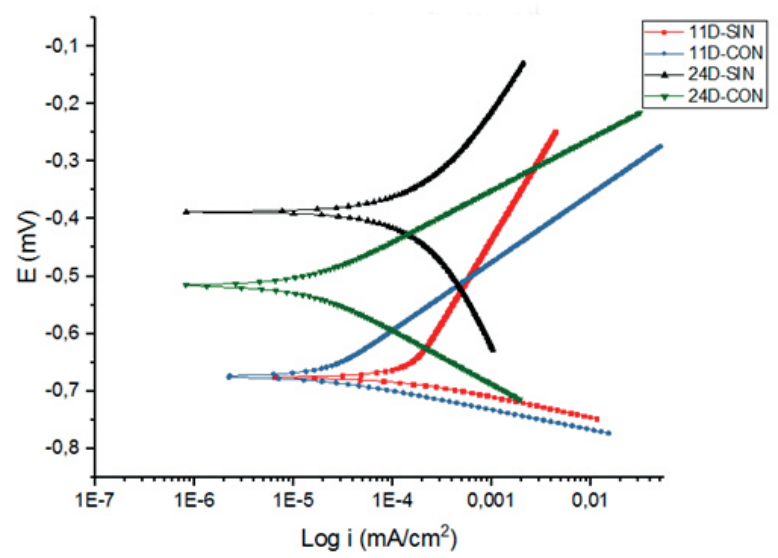

Figura 4. Curvas Tafel sometidas en $\mathrm{NaCl}$ y evaluadas en $\mathrm{NaCl}$ durante 24 días. las muestras sumergidas en medio ácido, y dejó áreas desprotegidas y con una mayor posibilidad de presentar zonas con diferencias de potencial que favorecieron el proceso de corrosión. Como se logró evidenciar en las micrografías obtenidas por medio de SEM, la capa protectora formada es más densa y homogénea en las muestras sumergidas en el medio salino en presencia del inhibidor que en el medio ácido en presencia del inhibidor.

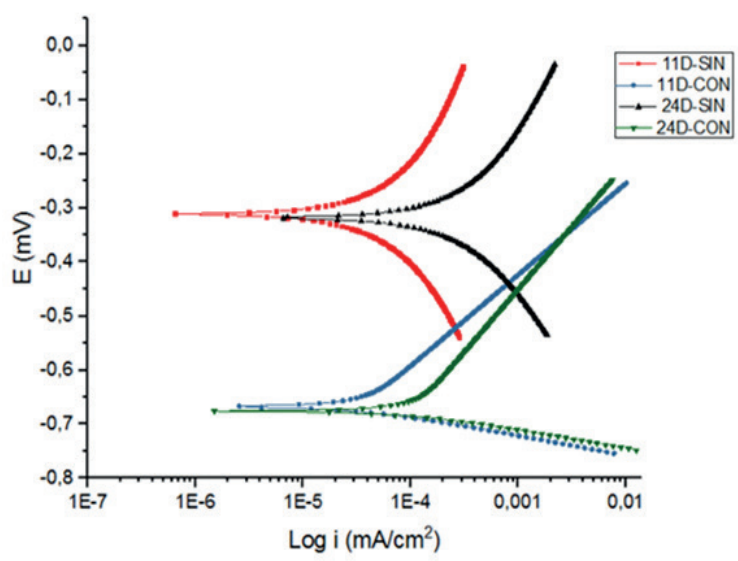

Figura 5. Curvas Tafel sometidas en $\mathrm{HCl}$ y evaluadas en agua destilada durante 24 días. 
Finalmente, fue calculada la eficiencia de inhibicion con ayuda de las velocidades de corrosión obtenidas a partir de las pendientes de las curvas Tafel, estos cálculos de eficiencia se realizaron para ambos medios. Se encontró que el inhibidor verde extraído de la cáscara de sandía que se utilizó en este estudio presenta una eficiencia del $94 \%$ (tabla 1) para el medio de $\mathrm{NaCl}$ durante los 24 día de exposición, lo cual indica que este inhibidor tiene potencial para ser utilizado como inhibidor de corrosión en el ámbito industrial en medios marinos [8]. Sin embargo, la eficiencia de inhibición para el caso del medio ácido no es tan notoria, pero sí aceptable para el mismo tiempo de exposición, ya que se alcanza un porcentaje del $77 \%$, sin embargo, el inhibidor logró disminuir la velocidad de corrosión en todos los casos estudiados como se evidencia en la figura 6 .

Tabla 1. Eficiencia del inhibidor en los medios evaluados $\mathrm{HCl}-\mathrm{NaCl}$.

\begin{tabular}{|c|c|c|}
\hline \multicolumn{3}{|c|}{$\begin{array}{c}\text { Velocidad de corrosion (mpy) - } 24 \text { días } \\
\text { Tafel - medio HCl }\end{array}$} \\
\hline Sin inhibidor & Con inhibidor & Eficiencia \\
\hline 67,66 & 15,7 & $77 \%$ \\
\hline \multicolumn{3}{|c|}{$\begin{array}{c}\text { Velocidad de corrosion (mpy) - } 24 \text { días } \\
\text { Tafel - medio } \mathrm{NaCl}\end{array}$} \\
\hline Sin inhibidor & Con inhibidor & Eficiencia \\
\hline 39,67 & 2,198 & $94 \%$ \\
\hline
\end{tabular}

\section{Conclusiones}

Se concluye experimentalmente que el inhibidor de cáscara de sandía proporciona una protección anódica contra la corrosión, mediante una película pasiva que aísla el metal del medio.

Se encontró que este inhibidor verde, en medio salino a una concentración de $\mathrm{NaCl}$ de 3,5\%, durante 24 días de exposición, presenta una eficiencia de $94 \%$, por lo cual es posible recomendarlo para ser utilizado en acero estructural A36 bajo estas condiciones. El inhibidor de cáscara de sandía en medio ácido, para 24 días de exposición tuvo eficiencias del $77 \%$, la cual resulta inferior al marco de referencia aceptable para recomendar un inhibidor que corresponda a eficiencias iguales o superiores al $85 \%$, por lo cual no es posible recomendar este inhibidor para acero A36 en condiciones ácidas.

\section{Referencias bibliográficas}

[1] Valladares-Cisneros MG, Melgoza-Alemán RM, Arteaga CC. Inhibidores naturales en el control de la corrosión de materiales metálicos. Inventio, la génesis de la cultura universitaria en Morelos. Inventio. 2015;11(25):37-41.

[2] Franco Tronco MI. Estudio electroquímico de las interacciones formadas por el extracto activo de las hojas de Morinda citrifolia en la inhibición de la corrosión de acero estructural (tesis de maestría). Toluca, México: Universidad Autónoma del Estado de México; 2017.

[3] Gomez Chacon VL. Eficiencia de un inhibidor verde extraído de cascara de manzana mediante el sistema SOCHLET en la corrosión del acero 1018 en medio ácido (tesis de maestría). Monterrey, México: Centro de Investigación en Materiales Avanzados; 2016.

[4] Benítez LPT, Castellar PJM, Percy EDA, Bravo MJB. Uso de extractos de plantas como inhibidores de corrosión. Inf. tec. 2014;78(2):155-64.

[5] Burgos NJ, Mollica AS, Navarro AM, Pastrana PP. Evaluación de stevia rebaudiana como inhibidor ecológico de la corrosión del acero al carbono en medio ácido (proyecto de pregrado). Mendoza, Argentina: Universidad Nacional de Cuyo; 2018.

[6] Saavedra-Navarrete LM, Hernández Ramírez F. Tecnología electroquímica y sus aplicaciones: inhibidores de corrosión a partir de residuos agroindustriales (proyecto de pregrado). Jalisco, México: Universidad Jesuita de Guadalajara; 2017.

[7] Berrocal Bravo MJ, Percy A, David E, Meza Castellar PJD. Evaluación de la eficacia inhibidora de corrosión del extracto acuoso de aguacate (tesis doctoral). Cartagena, Colombia: Universidad de Cartagena; 2013.

[8] Bravo MJB, Percy EDA. Evaluación de la eficiencia inhibidora de corrosión del extracto acuoso de aguacate (persea americana). Cartagena de Indias, Colombia: Universidad de Cartagena; 2013. 